\title{
Inhalt
}

\author{
Zum Inhalt - V
}

Haftungsausschluss - VI

1 Die unterschiedlichen Abwehrsysteme - 1

2 Die mechanischen und funktionellen Körperschranken - 5

$2.1 \quad$ Epithelien - 5

2.2 Binde- und Stützgewebe - 9

2.3 Kontrollierter Durchlass und Transport durch Epithelgewebe -13

3 Die angeborene Immunabwehr -17

3.1 Abwehrstoffe der Epithelien - 18

3.2 Wirkstoffe im Blut und in Gewebeflüssigkeiten - 20

3.2.1 Opsonine - 21

3.2.2 Faktoren des Komplementsystems - 23

3.2.3 Faktoren des Gerinnungssystems - 30

3.2.4 Faktoren des Kininsystems - 32

3.3 Mechanismen und Faktoren der zellulären Zusammenarbeit - 35

3.3.1 Zellrezeptoren und Zellaktivierung - 36

3.3.1.1 Phosphokinasen, ITAM, ITIM und Proteinphosphatasen - $\mathbf{3 8}$

3.3.1.2 Guanosinnukleotid-bindende Proteine - $\mathbf{4 0}$

3.3.1.3 Intranukleäre Rezeptoren - 41

3.3.2 Immunmediatoren (Zytokine) und Wachstumsfaktoren - 44

3.3.2.1 Interleukine -45

3.3.2.2 Chemokine -49

3.3.2.3 Interferone $-\mathbf{5 1}$

3.3.2.4 Faktoren für die Blutbildung (Hämatopoese) $-\mathbf{5 3}$

3.3.2.5 Tumor-Nekrose-Faktor-Familie - 56

3.3.2.6 Wachstumsfaktoren -60

3.3.3 Zytoplasmatische Signalübertragungswege -62

3.3.4 Weitere Gewebshormone, beteiligt an der Immunabwehr -79

3.3.4.1 Prostaglandine $\mathbf{-} \mathbf{8 0}$

3.3.4.2 Leukotriene $-\mathbf{8 2}$

3.3.4.3 Histamin -83

3.3.4.4 Serotonin - 85

3.3.5 Adhäsionsproteine für den Zellkontakt $-\mathbf{8 6}$

3.3.6 Gesteuerte Wanderung (Chemotaxie) durch Blutgefäßwände (Diapedese) und Gewebe - 90

3.3.6.1 Schrittfolgen -92

3.3.6.2 Beteiligte Enzyme - 93 
3.3.7 Wachstum und Zellteilung - 96

3.3.8 Kontrollierter Zelltod (Apoptose) - 101

3.4 Eigenschaften der beteiligten Zellen - 105

3.4.1 Mastzellen -105

3.4.2 Blutplättchen/Thrombozyten - 107

3.4.3 Fresszellen (Phagozyten) $\mathbf{- 1 1 0}$

3.4.3.1 Granulozyten - 111

3.4.3.2 Makrophagen - 117

3.4.4 Phagozytose und Exozytose — 122

3.4.4.1 Erkennen von Fremdsubstanzen durch Rezeptoren für pathogene Strukturmuster - 122

3.4.4.2 Abtötung von Infektionserregern durch reaktive Sauerstoff- und Stickstoffmoleküle - 128

3.4.4.3 Aufnahme (Phagozytose), Abtötung und Verdau in einer Verdauungsvakuole und die Exozytose — 134

3.5 Intrazelluläre Vermehrung von bakteriellen Infektionserregern — 137

3.6 Natürliche Killerzellen - $\mathbf{1 4 0}$

3.7 Die lokale Entzündung nach Erstkontakt - 144

3.7.1 Freisetzung von pro- und antiinflammatorischen Wirkstoffen - 144

3.7.2 Beteiligung von Endothelzellen - $\mathbf{1 4 8}$

3.7.3 Ablauf der Entzündung - 151

3.7.3.1 Gefäßneubildung (Angiogenese) zur Abgrenzung des Entzündungherdes - 152

3.7.3.2 Vernarbung und Epithelisierung - 159

4 Die erworbene Immunabwehr - 161

4.1 Struktur der beteiligten lymphatischen Gewebe — 161

4.1.1 Thymus 162

4.1.2 Milz 163

4.1.3 Lymphgefäßsystem und Lymphknoten — 164

4.1.4 Schleimhaut-assoziiertes lymphatisches Gewebe (MALT, GALT) und die Rolle von Epithelzellen — 167

4.2 Immunogene, Antigene, Epitope - 169

4.3 Antigen-bindende Moleküle und die Polymorphie, somatische

Rekombination und somatische Hypermutation ihrer variablen

Domänen - 170

4.4 Stufenförmige Entwicklung der erworbenen Immunabwehr -180

4.5 Präsentation von antigenen Peptiden und von Lipiden - 182

4.5.1 MHC-I 184

4.5.1.1 Struktur -184

4.5.1.2 Synthese und Beladung - $\mathbf{1 8 5}$

4.5.1.3 Überkreuz-Beladung — 187

4.5.1.4 Präsentation durch Kern-haltige Zellen - 188

4.5.2 $\quad$ MHC-II 189 


\subsubsection{Struktur -189}

4.5.2.2 Synthese und Beladung - 189

4.5.2.3 Präsentation durch dendritische Zellen und andere Antigen-präsentierende Zellen -190

4.5.3 CD1 196

4.5.3.1 Struktur 196

4.5.3.2 Synthese und Beladung - 197

4.5.3.3 Präsentation durch dendritische Zellen und andere Antigen-präsentierende Zellen 198

4.5.4 MHC-I-verwandte Strukturen - 199

4.5.4.1 MICA und MICB - 199

4.5.4.2 ULBP und Rae-1 Proteine -200

4.5.4.3 nFcR 201

4.6 T-Lymphozyten und ihre Rezeptoren — 201

4.6.1 T-Lymphozyten-Rezeptoren und akzessorische Moleküle — 202

4.6.1.1 T-Lymphozyten-Rezeptor - 202

4.6.1.2 Akzessorische Moleküle CD3 und Zeta - 204

4.6.2 Korezeptoren -208

4.6.2.1 Korezeptor CD4 $\mathbf{2 0 8}$

4.6.2.2 Korezeptor CD8 208

4.6.3 Kostimulatoren, Inhibitoren und Modulatoren - 209

4.6.3.1 Rezeptoren der CD28-Familie - $\mathbf{2 1 0}$

4.6.3.2 NKG2D 216

4.6.3.3 Mitglieder der TNF-Rezeptorsuperfamilie - 217

4.7 Reifung und positive/negative Selektionen der T-Lymphozyten im Thymus 221

4.8 Aktivierung naiver T-Lymphozyten $-\mathbf{2 2 7}$

4.8.1 Bildung einer immunologischen Synapse mit dendritischen Zellen - 228

4.8.2 Regulierung der Aktivierung - 232

4.8.3 Toleranz durch Ignoranz, Anergie und Deletionen - 236

4.9 Zytotoxische T-Lymphozyten - 238

4.9.1 Entwicklung von MHC-abhängigen zytotoxischen T-Lymphozyten - 238

4.9.2 Entwicklung von CD1-abhängigen zytotoxischen T-Lymphozyten (natürliche Killer T-Lymphozyten, NKT) $-\mathbf{2 4 0}$

4.9.3 Aktivierung und zytotoxische Reaktion - 241

4.10 T-Helfer-Lymphozyten — 247

4.10.1 CD4(+)-T-Helfer(1)-Lymphozyten - 249

4.10.2 CD4(+)-T-Helfer(2)-Lymphozyten - 250

4.10.3 CD4(+)-T-Helfer(17)-Lymphozyten - 252

$4.11 \quad$ Regulatorische T-Lymphozyten - 254

4.11.1 Natürliche und induzierte Tregs - 254

4.11.2 Wirkung von Interleukin-10 260

4.11.3 Wirkung von TGFbeta -262

4.12 Gedächtnis-T-Lymphozyten — 266 
4.13 Aktivierung von T-Lymphozyten durch Superantigene - 269

4.14 Antikörper als Ergebnis der Entwicklung von B-Lymphozyten zu Plasmazellen $-\mathbf{2 7 2}$

4.14.1 Struktur der Antikörper — 273

4.14.2 Primäre Funktion: Bindung an antigene Determinanten - 281

4.14.3 Sekundäre (Effektor-)Funktionen - 284

4.14.3.1 Übersicht -284

4.14.3.2 Bindung an Fc-Rezeptoren - 287

4.14.3.3 Transport von IgA, IgM und IgG durch Zellschranken - 291

4.14.3.4 Bildung von Immunkomplexen - 293

4.14.3.5 Bildung von Antikörpern gegen die variablen Teile von Antikörpern (Antiidiotyp-Antikörper) -296

4.14.3.6 Bildung von Antikörpern gegen konstante Teile von Antikörpern (Rheumafaktoren) - 297

4.14.3.7 Bindung von Komplementfaktoren und Aktivierung von KomplementRezeptoren - 298

4.14.3.8 Phagozytose, Exozytose und Entzündung - $\mathbf{3 0 5}$

4.14.3.9 Antikörper-vermittelte zytotoxische Reaktionen - 306

4.15 B-Lymphozyten und ihre Rezeptoren - $\mathbf{3 1 0}$

4.15.1 B-Lymphozyten-Rezeptor - $\mathbf{3 1 2}$

4.15.2 Stimulierende Korezeptoren und Kostimulatoren - 313

4.15.2.1 CD19 und sein Komplex mit CD21, CD81 und CD225 - 313

4.15.2.2 Aktivierende FC-Rezeptoren - 314

4.15.2.3 TLR und CD180 im Komplex mit MD-1 - 315

4.15.2.4 Mitglieder der TNF-Rezeptorsuperfamilie - 315

4.15.3 Koinhibitoren - 319

4.15.3.1 Fc-gamma-Rezeptor-IIB - 319

4.15.3.2 Leukozyten-Immunoglobulin-ähnliche Rezeptoren-Subfamilie B - $\mathbf{3 1 9}$

4.15.3.3 CD22-B-Lymphozyten Antigen — 319

4.15.3.4 CD72-Antigen und seine Liganden CD100 und CD5 - $\mathbf{3 2 0}$

4.15.3.5 CD30 (TNF-Rezeptorsuperfamilie) und sein Ligand - $\mathbf{3 2 1}$

4.15.4 Rezeptoren, welche fördernd wie auch hemmend wirken können - 322

4.15.4.1 Fc-epsilonRIla (CD23a) - 322

4.15.4.2 Fc-Rezeptor-Homologe - 323

4.15.5 Weitere Liganden und Adhäsionsmoleküle - 324

4.15.5.1 CD80/CD86-324

4.15.5.2 ICOS-Ligand (B7-H2) - 325

4.15.5.3 CD134-Ligand (Ox40L, Gp34) - 326

4.15.5.4 Adhäsionsmoleküle - 326

4.16 Kooperation zwischen B-Lymphozyten und T-Helfer(2)-Lymphozyten - 328

4.16.1 Aufnahme von Fremdsubstanzen und Antigen-Präsentation durch

B-Lymphozyten - 328

4.16.2 Bildung einer immunologischen Synapse mit CD4(+)-T-Helfer(2)Lymphozyten - 331 
4.16.3 Hilfe durch Zytokine der CD4(+)-T-Helfer(2)-Lymphozyten — 334

4.17 Antigen-abhängige Reifung von B-Lymphozyten — 335

4.17.1 Entwickung und Selektion von virginellen (naiven) B-Lymphozyten - 335

4.17.2 Entwicklung und Selektion von follikulären B-Lymphozyten, Kooperation mit T-Helfer-Lymphozyten - 336

4.17.3 Bildung von Zentroblasten (Zellstadium der somatische Hypermutation) - 337

4.17.4 Bildung von Zentrozyten und Selektion auf Bildung hochaffiner Antikörper (Affinitätsreifung) - 339

4.17.5 Synthese von IgM und Wechsel der Antikörperklasse (Isotyp-Switch) - $\mathbf{3 4 0}$

4.17.5.1 Synthese der schweren (H-)Kette von IgM - 340

4.17.5.2 Synthese der leichten (L-)Ketten - 342

4.17.5.3 Wechsel der Antikörperklasse (Isotyp-Switch) - 344

4.17.5.4 Zusammenfügung des Antikörpermoleküls und dessen Expression — 346

4.17.6 Entwicklung zu Plasmazellen - 347

4.17.7 Entwicklung zu Gedächtnis-B-Lymphozyten - $\mathbf{3 4 9}$

4.18 Aktivierung von B-Lymphozyten durch T-Lymphozyten-unabhängige Antigene -351

4.19 Steuerung der Synthese und des Abbaus von Antikörpern - 353

$5 \quad$ Der Einfluss des Nervensystems auf das Immunsystem - 359

5.1 Verbindendes zwischen beiden Organsystemen - 359

5.1.1 Experimentelle und klinische Hinweise - 359

5.1.2 Funktionelle Verbindungen - $\mathbf{3 5 9}$

5.1.3 Rolle der Nervenzellen - 361

5.1.4 Rolle der Gliazellen - $\mathbf{3 6 5}$

5.1.4.1 Makroglia -365

5.1.4.2 Mikroglia - 366

5.1.4.3 Schwann'sche Zellen - 367

5.1.5 Rolle des Hypothalamus -369

$5.2 \quad$ Blut-Hirn-Schranke - $\mathbf{3 7 2}$

5.2.1 Struktur - $\mathbf{3 7 2}$

5.2.2 Durchlässigkeit - 373

5.2.3 Aufhebung der Barriere - 374

$5.3 \quad$ Blut-Liquor-Schranke - 376

5.4 Das Nervensystem als Steuerungszentrale der Immunabwehr — 377

5.4.1 Neurotransmitter des vegetativen Nervensystems - $\mathbf{3 8 1}$

5.4.1.1 Katecholamine und das adrenerge/dopaminerge System — 381

5.4.1.2 Acetylcholin und das cholinerge System - 384

5.4.1.3 Gleichgewicht der Wirkungen des adrenergen und des cholinergen Systems auf die Immunabwehr - $\mathbf{3 8 8}$

5.4.2 Neuropeptide $-\mathbf{3 8 9}$

5.4.2.1 Neuropeptid $Y-389$

5.4.2.2 Tachykinine -391 
5.4.2.3 Endorphine und Enkephaline - 392

5.4.2.4 CGRP -394

5.4.2.5 VIP und PACAP — 396

5.4.2.6 Natriuretische Peptide - 398

5.4.2.7 Ausgewogenheit der Wirkungen der Neuropeptide auf die Immunabwehr - 401

5.4.3 Hormone des Hypothalamus/der Neurohypohyse

(Hypophysenhinterlappen) $-\mathbf{4 0 4}$

5.4.3.1 Oxytocin -404

5.4.3.2 Arginin-Vasopressin $-\mathbf{4 0 6}$

5.4.4 Liberine und Statine des Hypothalamus - 409

5.4.4.1 Corticoliberin -409

5.4.4.2 Thyreoliberin $-\mathbf{4 1 2}$

5.4.4.3 Somatoliberin und Somatostatin - 412

5.4.4.4 Gonadoliberin - 416

5.4.4.5 Prolaktoliberin und Dopamin - 417

5.4.4.6 Melanoliberin und Melanostatin - 418

5.4.5 Hormone des Hypophysenvorderlappens und Hypophysenzwischenlappens - 419

5.4.5.1 Adrenocorticotropin - 419

5.4.5.2 Wachstumshormon $-\mathbf{4 2 2}$

5.4.5.3 Thyreotropin -423

5.4.5.4 Follitropin, SPH -424

5.4.5.5 Lutropin, Choriongonadotropin, LSH -425

5.4.5.6 Prolaktin -426

5.4.5.7 Melanotropin $-\mathbf{4 2 8}$

5.4.6 Hormone der Nebennierenrinde - 432

5.4.6.1 Glucocorticoide - 432

5.4.6.2 Mineralocorticoide -437

5.4.7 Sexualsteroide -439

5.4.7.1 Östrogene $-\mathbf{4 4 2}$

5.4.7.2 Progesteron $-\mathbf{4 4 5}$

5.4.7.3 Androgene $-\mathbf{4} 48$

5.4.8 Schildrüsenhormone $-\mathbf{4 5 0}$

5.5 Das Immunsystem als Signalgeber für das Nervensystem — 453

5.5.1 Immunzellen als Produzenten von Wirkstoffen des Nervensystems - 453

5.5.2 Immunmediatoren als Neuropeptide -456

5.5.3 Immunmediatoren bei der Entstehung von Schmerzen — 456

6 Herausforderungen und Fehlentwicklungen der Immunabwehr -463

6.1 Toleranz der Immunabwehr — 463

6.1.1 Entwicklung der zentralen Toleranz — 464

6.1.1.1 Selektion von Thymozyten - 464

6.1.1.2 Selektion von B-Lymphozyten — 465 
6.1.2 Aufrechterhaltung der Toleranz in der Peripherie - $\mathbf{4 6 6}$

6.1.2.1 Ignoranz, Deletion und Anergie von T-Lymphozyten - 466

6.1.2.2 Mangelnde Hilfe für B-Lymphozyten und zytotoxische

T-Lymphozyten - 467

6.1.2.3 Suppression von T-Helfer-Lymphozyten durch regulatorische T-Lymphozyten - $\mathbf{4 6 8}$

6.1.2.4 Hemmung von natürlichen Killerzellen - 469

6.1.2.5 Homöostase -469

6.1.2.6 Einfluss von Neurotransmittern, Neuropeptiden und Hormonen $-\mathbf{4 7 0}$

6.1.3 Toleranz des Fetus während der Schwangerschaft $\mathbf{- 4 7 2}$

6.1.3.1 Fehlende Antigen-Präsentation (MHC-II, MHC-Ia) und Expression von MHC-Ib (HLA-G) -473

6.1.3.2 Induktion von Apoptose durch FAS-Liganden -474

6.1.3.3 Inhibition der Komplementaktivierung durch MCP (CD46), DAF (CD55) und HRF (CD59) - 475

6.1.3.4 Einfluss von Progesteron und des Prosteron-induzierten blockierenden Faktors -475

6.1.3.5 Expression des Leukämie-inhibierenden Faktors — 477

6.2 Unverträglichkeiten zwischen Blutgruppen - 479

6.2.1 Blutgruppen A, B, AB, $0-481$

6.2.2 Rhesusfaktoren $-\mathbf{4 8 2}$

6.2.3 Kell-Cellano-Antigene $-\mathbf{4 8 4}$

6.2.4 Duffy-Antigene - 486

6.2.5 Kidd-Antigene $-\mathbf{4 8 8}$

6.2.6 MNS-Blutgruppe $-\mathbf{4 9 0}$

6.3 Immunschwächen $-\mathbf{4 9 2}$

6.3.1 Angeborene (primäre) Immundefekte — 492

6.3.2 Erworbene (sekundäre) Immundefizienzen — 499

6.3.2.1 Immunschwäche des Neugeborenen — 501

6.3.2.2 Alterung der Immunabwehr - $\mathbf{5 0 7}$

6.4 Schlaf, Belastungen und Stress - $\mathbf{5 1 0}$

6.4.1 Schlaf und Erholung - $\mathbf{5 1 0}$

6.4.2 Körperliche Tätigkeiten $-\mathbf{5 1 3}$

6.4.3 Stress $\mathbf{5 1 6}$

6.5 Abwehr von Infektionserregern - $\mathbf{5 1 9}$

6.5.1 Bakterieninfektionen - $\mathbf{5 2 0}$

6.5.1.1 Pathogene Gram(+)-Bakterien - $\mathbf{5 2 4}$

6.5.1.2 Pathogene Gram(-)-Bakterien, Endotoxin (LPS) und CD14 532

6.5.2 Virusinfektionen $-\mathbf{5 4 1}$

6.5.3 Pilzinfektionen $\mathbf{- 5 4 8}$

6.5.4 Parasiteninfektionen - $\mathbf{5 5 1}$

6.6 Systemisches Immunreaktionssyndrom und Sepsis - $\mathbf{5 6 2}$

6.7 Allergische Reaktionen - $\mathbf{5 7 1}$

6.7.1 Typ I: allergische Reaktionen vom Soforttyp und Pseudoallergien — $\mathbf{5 7 3}$ 
6.7.1.1 Akute Phase (Einfluss der Mastzellen und basophilen Granulozyten) - $\mathbf{5 7 3}$

6.7.1.2 Chronische Phase (Einfluss der eosinophilen Granulozyten) - $\mathbf{5 8 2}$

6.7.1.3 Beeinflussung durch endogene und exogene Faktoren - $\mathbf{5 8 5}$

6.7.1.4 Der allergische Formenkreis und dessen Behandlung — 596

6.7.2 Typ II: Antikörper-vermittelte allergische Reaktionen gegen Zell-gebundene Antigene -605

6.7.2.1 Übersicht -605

6.7.2.2 Autoimmunhämolytische Anämien — 606

6.7.2.3 Autoimmunthrombozytopenische Purpura - 608

6.7.2.4 Autoimmunneutropenien - 611

6.7.3 Typ III: allergische Reaktionen durch Immunkomplexe - $\mathbf{6 1 3}$

6.7.3.1 Mechanismen - 613

6.7.3.2 Gefäßentzündungen durch Immunkomplexe - $\mathbf{6 1 6}$

6.7.3.3 Gefäßentzündungen durch Antikörper gegen Granulozyten oder Endothelzellen - 617

6.7.3.4 Therapeutische Maßnahmen - 620

6.7.4 Typ IV: allergische Reaktionen vom verzögerten Typ - $\mathbf{6 2 1}$

6.7.4.1 Durch CD4(+)-T-Helfer(1)-Lymphozyten und Makrophagen - 622

6.7.4.2 Durch zytotoxische CD8(+)-T-Lymphozyten oder durch eosinophile Granulozyten - 626

6.7.5 Allergische Reaktionen gegen Arzneimittel - 629

6.8 Autoimmunerkrankungen - 631

6.8.1 Häufigkeit, endogene und exogene Einflussfaktoren - 631

6.8.2 Autoimmunerkrankungen - vorwiegend des Nervensystems - 639

6.8.3 Autoimmunerkrankungen - vorwiegend endokriner Organe - 641

6.8.4 Autoimmunerkrankungen - vorwiegend der Haut - 642

6.8.5 Autoimmunerkrankungen - vorwiegend der Gelenke und der Muskulatur — 647

6.8.6 Autoimmunerkrankungen - vorwiegend von Leber, Pankreas, Niere und Lunge, Magen und Darm - 652

6.8.7 Autoimmunerkrankungen - vorwiegend durch Antikörper gegen Bestandteile des Blutes - 658

6.8.8 Autoimmunerkrankungen - vorwiegend der Blutgefäße und des Herzens -659

6.8.9 Kollagenosen - 661

6.8.10 Entzündungen des Auges bei Autoimmunerkrankungen - 663

6.8.11 Therapiemöglichkeiten -668

6.9 Abwehr von Tumoren - 670

6.9.1 Tumorerkrankungen als Ergebnis mangelhafter Immunabwehr $-\mathbf{6 7 0}$

6.9.2 Tumor-spezifische und tumor-assoziierte Antigene - 674

6.9.3 Tumorimmundiagnostik -689

6.9.4 Tumorimmuntherapie -694

6.9.4.1 Hintergrund - 694

6.9.4.2 Monoklonale Antikörper — 697 


\subsubsection{Zytokine - 702}

6.9.4.4 Impfstoffe und Vakzinen - 704

6.10 Transplantationen von Geweben und Organen - 709

6.10.1 Abstoßungsreaktionen - 709

6.10.2 Transplantation von hämatopoetischen Stammzellen -714

$7 \quad$ Arzneimittel und Zubereitungen zur Beeinflussung der Immunabwehr -717

7.1 Modulation der Immunabwehr — 717

7.1.1 Aktive Antigen-spezifische Stimulierung/Schutzimpfungen - $\mathbf{7 1 8}$

7.1.1.1 Impfstoffe gegen Viren und Bakterien - $\mathbf{7 2 0}$

7.1.1.2 Impfstoffe gegen Pilze und Parasiten - 728

7.1.2 Passive Antigen-spezifische Stimulierung und Modulierung - 730

7.1.2.1 Polyklonale Antikörper aus dem Blut und deren Spaltprodukte -730

7.1.2.2 Monoklonale Antikörper, rekombinante Antikörper und Fusionsproteine - 738

7.1.3 Antigen-unspezifische Modulierung der Immunabwehr — 746

7.1.3.1 Aktiv mit Immunstimulantien - 746

7.1.3.2 Passiv mit Zytokinen und Fusionsproteinen - $\mathbf{7 4 8}$

7.2 Hemmung der Immunabwehr durch Immunsuppressiva - 752

$8 \quad$ Anlagen -761

8.1 Hinweise zu weiteren Informationen — 761

8.1.1 CD-Nomenklatur - 761

8.1.2 Weiterführende Literatur — 761

8.1.3 Biochemische und pharmakodynamische Charakteristika von Molekülen der Immunabwehr -761

8.1.4 Impfstoffe, monoklonale Antikörper, Immunglobulinpräparate, Immunmediatoren, Allergene für die Desensibilisierung — 761

8.1.5 Leitlinien für die Behandlung von immunologischen Erkrankungen - 762

\section{Sachregister -763}

Über den Autor — 791 
